\title{
Ecosystem self-organization in the Venice Lagoon
}

\author{
G. Cecconi ${ }^{1}$, C. Cerasuolo ${ }^{1}$, E. Del Giudice ${ }^{2,3}$, N. Marchettini ${ }^{4}$ \\ \& E. Tiezzi ${ }^{4}$ \\ ${ }^{1}$ Consorzio Venezia Nuova, Italy \\ ${ }^{2}$ International Institute of Biophysics, Germany \\ ${ }^{3}$ INFN, Italy \\ ${ }^{4}$ Department of Chemistry, University of Siena, Italy
}

\begin{abstract}
The formation of salt marshes (barene) in the Venice Lagoon is discussed in light of recent theories about the emergence of novelties. Namely, the emergent properties and the new formations are related to field quantum coherence domains and to dissipative structures.

The boundary conditions, the ontic openness of the ecosystem and the continuous flow of energy and materials allow self-organization during the salt marshes formation and development.

The role of the interphase and of the increasing mud deposit is also discussed from the point of view of Prigogine's Thermodynamics, taking into account the constraints and the limit conditions.
\end{abstract}

Keywords: salt marshes, self-organization, Venice Lagoon, coherence-domains.

\section{Introduction}

In the last few years the observation of the evolution occurring in the Venice Lagoon near some artificial sediment confinement has allowed light to be shed on the process of formation of tidal ecosystems. In the present paper we summarize such observations and try to outline a conceptual frame where such observations can find a rationale. A short preliminary résumé has been presented elsewhere [1]. 
The Venice Lagoon in the last decades has been plagued by two kinds of disruption:

i. chemical wastes coming both from chemical plants, urban settlement and agricultural land have reduced biodiversity and structural selfconservation capabilities;

ii. the deepening of tidal flats produced by sea level rise, subsidence and the excavation of artificial channels have modified the hydro-morphological flow field, increasing the wind-wave energy and inducing potentially disruptive imbalances.

In order to counter these disruptive dynamics, artificial salt marsh and intertidal flats have been designed and constructed. A surprising result has been observed to have arisen 3-5 years after the start of the above works.

Salt-marshes habitat (barene) have started to appear (see Figures 1-4) at an accelerated pace above the sediment deposits in the protected area. Meanwhile, in the other untreated areas of the lagoon, this process is occurring only in peculiar limited places.

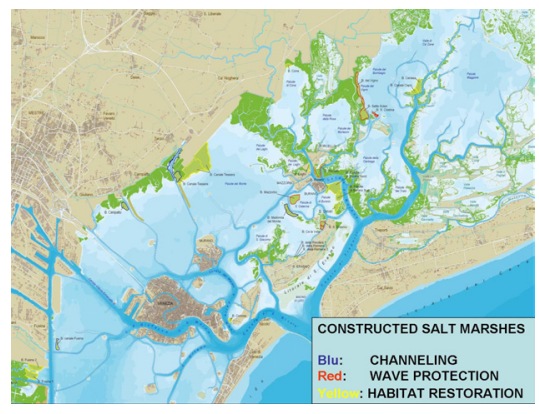

Figure 1: Constructed salt marshes re-using sediments from channel maintenance dredging in the Northern lagoon (a).

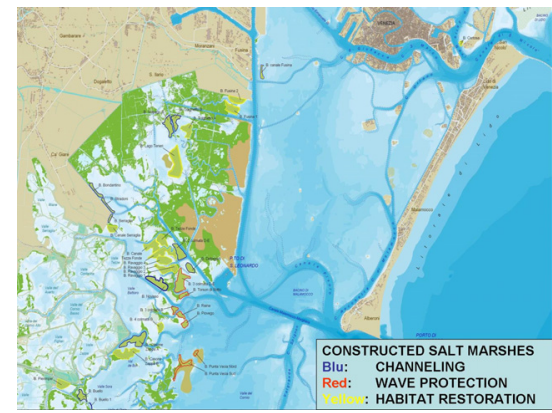

Figure 2: Constructed salt marshes re-using sediments from channel maintenance dredging in the central lagoon (b). 


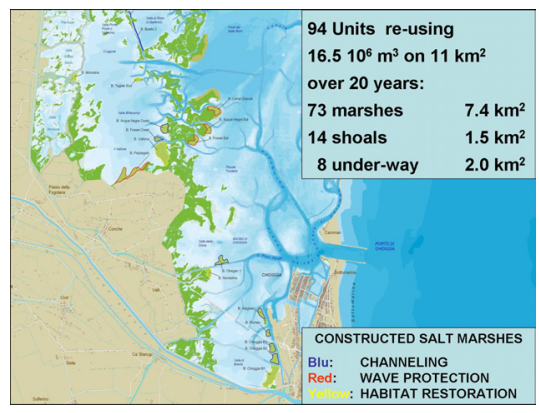

Figure 3: $\quad$ Dec. 2008 total constructed salt marshes re-using sediments from channel maintenance dredging in the Southern lagoon (c).

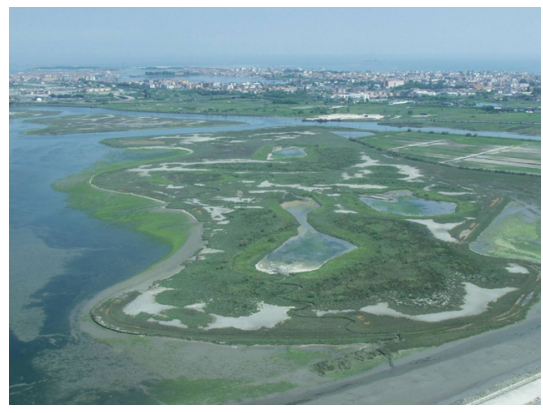

Figure 4: Constructed salt marshes in Chioggia after 10 years.

Vigorous wetland life has erupted and developed intensely both ways [2].

Similar results have been obtained in the artificial reef constructed for the protection of the new inlet breakwaters at the Mose Flood Barriers [3].

How has that been possible?

\section{The conceptual frame}

Recent developments in scientific thinking, in particular Thermodynamics of Irreversible Processes (TIP) and Quantum Field Theory (QFT) could help us to get an understanding of what is going on.

In a recent paper [4] it has been pointed out how the interplay of TIP and QFT could be at the root of ecosystem dynamics. Let us summarize what is the suggested dynamics. It is a general feature in QFT that an ensemble of $\mathrm{N}$ oscillators coupled with a mode of the electromagnetic (e.m.) field having a wavelength $\lambda$ starts to oscillate in unison (this is the coherence) in phase with 
the e.m. field when the density $\frac{N}{\lambda^{3}}$ of the oscillators exceeds a critical threshold and the temperature $\mathrm{T}$ lies below a critical value. In this way an extended region (coherence domain C.D., [5]) is formed where order appears spontaneously; all molecules move coherently on a well defined degrees of freedom, concentrating on it the whole available energy, so leaving the other degree of freedom empty. This process of concentration is made possible by the fact that the coherent state has an energy lower than the original non-coherent state. In this way energy is released outwards, increasing the entropy $S_{e}$ of the environment, whereas the internal entropy $S_{i}$ of the $C D$ sharply decreases. The Second Law of Thermodynamics is satisfied since, as outlined in Tiezzi [6]:

$$
\Delta S_{e}+\Delta S_{i} \geq 0
$$

in spite of the decrease of internal entropy

$$
\Delta S_{i}<0
$$

It has been shown in Arani et al [7] that the liquid state emerges, in welldefined thermodynamical conditions, from the gaseous non-ordered phase. In a liquid coherence involves the electron degrees of freedom, whereas the solid state corresponds to the onset of coherence among nuclei. Coming back to the liquids, water exhibits a unique property. In the water CDs the coherent oscillation of molecules occurs between the following two configurations [7]:

i. the ground configuration where all electrons are tightly bound;

ii. the excited configuration $(12.06 \mathrm{eV})$ where one electron is just below the ionization threshold $(12.60 \mathrm{eV})$.

As a consequence, in the $\mathrm{CD}$ of water there is permanently a reservoir of almost free electrons that are able to perform two tasks:

a) they can release electrons outwards, thus supplying external oxidizing molecules. In this way water CDs become the reducing partners in the redox reactions [8]. The surface separating the $\mathrm{CD}$ from the non-coherent surroundings becomes thus a redox pile of about one volt;

b) an inflow of external energy can induce a vortex in the ensemble of almost free electrons, whose angular momentum is quantized and is allowed to assume only integer values. Since the motion of the electrons in the vortex is coherent, collisions cannot occur so that the attrition is vanishing. As a consequence, the lifetime of these vortices is extremely long. It is then possible to: iterate the excitations producing a pile-up of superimposed vortices. The water CDs, and this is a unique feature of water, are able to collect in the environment low-grade energy in incoherent form (high entropy) and transform it into high-grade energy (low entropy) able to induce electronic excitations of molecules [8,9]. 
When the energy stored in the CDs matches the activation energy of neighboring molecules, it is transferred instantly by resonance; molecules get activated and the CD discharges itself, becoming ready for a new cycle.

Water CDs, through the above interplay of electromagnetism, chemistry and thermodynamics, become in turn oscillators and the general theorem of QFT stated above allows them to become coherent in turn, producing extended coherence domains made up of coherence domains $[4,8,9]$, whose rhythm of oscillation is determined by the combination of the rate of energy loading (controlled by the intensity of the energy inflow) and the rate of energy discharge (controlled by the value of the activation energy of the neighboring molecules). The size of the coherent region corresponds to the wavelength of the e.m. mode corresponding to the above rhythm of oscillation. In this way the whole system made up of water, interacting molecules, flows of energy and interfaces becomes able to self-organize, according to a scheme fulfilling the requirements of TIP as stated by Prigogine and Nicolis [10].

In the above scheme it is essential the role of the interfaces. Actually water molecules, left alone, would be subjected to the opposite dynamics of the electrodynamic attraction induced by the coherence (what in the Chinese philosophy is the yin) and the disruptive effect of the collisions (the yang), so that in bulk water the coherent structure is flickering, oscillating continuously between coherence and non-coherence.

Near a hydrophilic surface the attraction between water and wall stabilizes the coherent structure, shielding it against the effect of thermal collisions. Water near the interfaces becomes then permanently coherent (exclusion zone found by the Pollack group [11]) and is able to produce the long-term dynamics described above that brings to self-organization. Liquid water is able to exhibit its selforganizing capabilities only if is protected from too violent external stimuli, such as temperature or, at a macroscopic level, turbulence. The conceptual frame outlined here appears a good candidate to understand the patterns observed in the Venice Lagoon.

\section{Results and discussions}

The construction works by Magistrato alle Acque - Consorzio Venezia Nuova gives rise to the formation of new salt marshes (barene): emergence of novelties due to the creation of a confined intertidal soil interface.

The new soil surface relatively protected allow biodiversity to increase from successions of soil improvements, alofitic vegetation, bird and fish species (see Figures 5 and 6).

This evolution is related to the idea of confined-ontic-openness [12]. These ecosystems are open thermodynamic systems exchanging energy and matter with the outside (the lagoon) due to tidal transport that is still possible through the partial artificial confinement. 


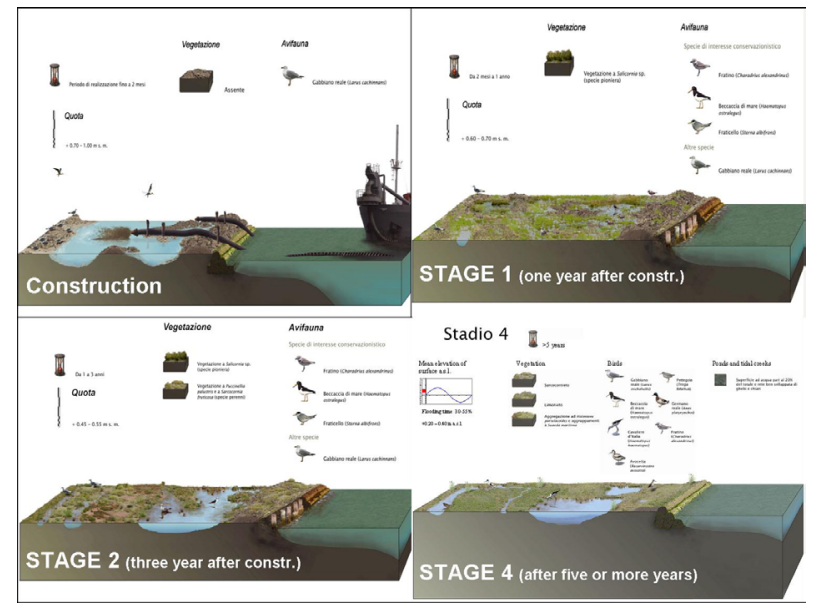

Figure 5: The increasing of biodiversity in the artificial salt marshes as a stage transitions.

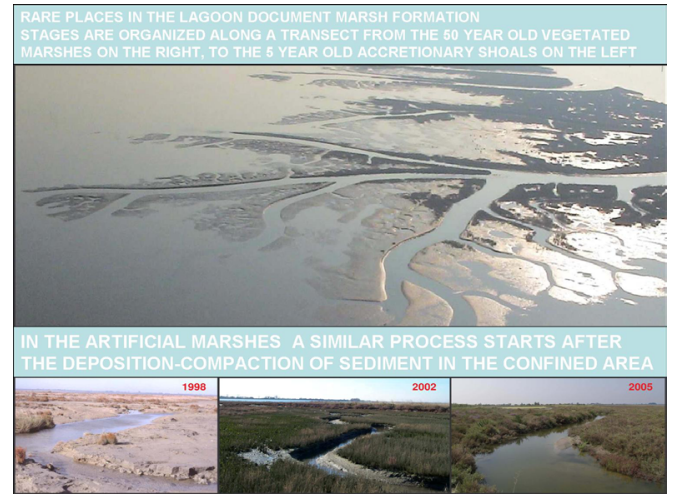

Figure 6: Marsh stages and self-organization: in a natural sheltered area (above) and in an artificial marsh (below).

They behave as Prigogine's dissipative structures. Entropy reaches a maximum (thermal death and thermodynamic equilibrium) in two cases:

a) an isolate system (no exchange);

b) an open system where too many exchanges and excess of turbulent energy dissipation lead to homologation (Clausius) and finally to the thermal death: no more differences.

The same argument of the salt-marshes (confined open systems) can be applied to the creation and first civilization of a town: e.g. Venice a port open to 
fluxes of trading resources but protected by the lagoon waters and Siena, protected by the Middle-Ages walls.

We may call this phenomenon: City out of Chaos [13], from the Prigogine's statement "Order out of Chaos".

\section{Conclusions}

In conclusion, we can say that two widely different entities, the salt marshes of the Venice Lagoon and the above mentioned Middle Age cities, share some common patterns that are the consequence of the very dynamics of selforganization.

\section{References}

[1] Cecconi, G., Cerasuolo, C., Marchettini, N., Tiezzi, E., Salt-marshes as emergent novelties in the Venice Lagoon. Ecological Modelling, special issue, 2009, in press.

[2] Cecconi, G., Cerasuolo, C., Turco F., Naturalizzazione delle barene artificiali. Accademia Nazionale dei Lincei La salvaguardia di Venezia e della sua laguna, Roma, 5 giugno 2008.

[3] Cecconi G., Cerasuolo C., Curiel D., Riccato F., Rismondo A., Rosa Salva P., Torricelli P., Nuovi habitat costieri dalle opere per la difesa dal mare. Gli ambienti sommersi delle scogliere di Pellestrina e Malamocco. Quaderno Trimestrale del Consorzio Venezia Nuova, 1.08, pp. 11-35, 2008.

[4] Del Giudice, E., Pulselli, R.M., Tiezzi, E., Thermodynamics of irreversible processes and Quantum Field Theory: an interplay for the understanding of ecosystem dynamics. Ecological Modelling, special issue, 2009, in press.

[5] Preparata, G., QED Coherence in Matter, World Scientific, London, 1995.

[6] Tiezzi, E., The Essence of Time, WIT press, Southampton, 123 pp., 2003.

[7] Arani, R., Bono, I., Del Giudice, E., Preparata, G., QED Coherence and the Thermodynamics of Water. Int J Mod Phys B, 9, pp. 1813, 1995.

[8] Del Giudice, E., Tedeschi, A., Water and autocatalysis in living organisms. Electromagnetic Biology and Medicine, 2009, in press.

[9] Voeikov, V. L., Del Giudice, E., Water respiration. Water (e-journal), in press, 2009.

[10] Prigogine, I., Nicolis, G., Self-Organization in Non-Equilibrium Systems, Wiley, New York, 1977.

[11] Zheng, J.-M., Chin, W.-C., Khijniak E., Khijniak E., Jr., Pollack, G. H., Surfaces and interfacial water: Evidence that hydrophilic surfaces have long-range impact. Adv Colloid Interface Sci, 127, pp. 19-27, 2006.

[12] Jørgensen, S. E., Fath, B. D., Bastianoni, S., Marques, J. C., Müller, F., Nielsen, S. N., Patten, B. C., Tiezzi, E., Ulanowicz, R. E., A New Ecology: Systems Perspective, Elsevier, Amsterdam, 288 pp, 2007.

[13] Pulselli, R.M., Tiezzi, E., City out of chaos, WIT press, Southampton, 176 pp., 2009. 